Supporting Information for

\title{
Thermodynamic Analysis of $\mathrm{LiNi}_{0.6} \mathrm{Mn}_{0.2} \mathrm{Co}_{0.2} \mathrm{O}_{2}$ (NMC622) Voltage Hysteresis Induced through High Voltage Charge
}

Wenzao Li ${ }^{\text {a,b }}$, Lisa M. Housel ${ }^{\text {b,c }}$, Garrett P. Wheeler ${ }^{\text {b,c }}$, David C. Bock b,c Kenneth J. Takeuchi ${ }^{a, b, c, d}$, Esther S. Takeuchi ${ }^{a, b, c, d}$, and Amy C. Marschilok ${ }^{a, b, c, d^{*}}$

a Department of Chemistry, State University of New York at Stony Brook, Stony Brook, New York 11794, United States;

b Institute for Electrochemically Stored Energy, Stony Brook University, Stony Brook, New York 11794, United States;

c Interdisciplinary Science Department, Brookhaven National Laboratory, Upton, New York 11973, United States;

d Department of Materials Science and Chemical Engineering, State University of New York at Stony Brook, Stony Brook, New York 11794, United States.

\section{Contents}

1 Experimental Section $\quad$ S-2

1.1 Materials characterizations . . . . . . . . . . . . . . S -2

1.2 Electrode preparation . . . . . . . . . . . . . . . S S-2

1.3 Electrochemistry . . . . . . . . . . . . . . . . . . S-2

1.4 Operando isothermal microcalorimetry $(\mathrm{IMC}) \ldots \ldots \ldots$. . . . . . . -2

2 Theory S-2

2.1 Introduction . . . . . . . . . . . . . . . . . . . S-2

2.2 Equations . . . . . . . . . . . . . . . . . . . . . . . . . . .

2.3 Discussion . . . . . . . . . . . . . . . . . . . S-5

3 Supplemental Results S-6

3.1 The characterization of NMC622 electrodes . . . . . . . . . . . . S-6

3.2 Electrochemistry of formation cycles . . . . . . . . . . . . . S-7

3.3 The duplicate IMC tests . . . . . . . . . . . . . . . . . . . . . S-8

3.4 Operando IMC tests: Electrochemistry and real-time heat flow . . . . S-9

3.5 The estimation of thermodynamic equilibrium potential by GITT . . . S-11

3.6 Potentiometric measurement of entropy . . . . . . . . . . . . . . . S-12

3.7 Electrochemistry shown as chemical composition . . . . . . . . . . S S-12

3.8 Normalized heat generated per unit of capacity . . . . . . . . . . . S -13

4 References $\quad$ S-13

*Corresponding Email: amy.marschilok@stonybrook.edu 


\section{Experimental Section}

\subsection{Materials characterizations}

Scanning electron microscopy (SEM) images were collected on a JEOL JSM6010 Plus/LA microscope with an accelerating potential of $5 \mathrm{kV}$.

\subsection{Electrode preparation}

NMC electrodes were prepared with a 90:5:5 mass ratio of $\mathrm{LiNi}_{0.6} \mathrm{Mn}_{0.2} \mathrm{Co}_{0.2} \mathrm{O}_{2}$ (NMC622, Targray, 9-18 $\mu \mathrm{m}$ particle size), Super P carbon (TimCal, $40 \mathrm{~nm}$ particle size), and polyvinylidene fluoride binder (Kynar). The composite slurry was cast onto carbon-coated aluminum foil using N-methyl-2-pyrrolidone solvent and then dried. The NMC electrode loadings were $11.5 \mathrm{mg} / \mathrm{cm}^{2}$.

\subsection{Electrochemistry}

2032-type coin cells were assembled using NMC622 composite electrodes as the cathodes, lithium metal foils as anodes, and $1 \mathrm{M} \mathrm{LiPF}_{6}$ in 3:7 ethylene carbonate (EC)/dimethyl carbonate electrolyte (DMC) as the electrolyte in an argon-filled glovebox. Formation cycles and Galvanostatic Intermittent Titration Technique (GITT) tests were performed by galvanostatic cycling on a MACCOR series 4000 battery testing system at $30{ }^{\circ} \mathrm{C}$. For formation cycling, coin cells were cycled 5 times: the first 4 cycles over $3-4.3 \mathrm{~V}$ (vs. $\mathrm{Li} / \mathrm{Li}^{+}$) followed by the $5^{\text {th }}$ cycle over either $3-4.3 \mathrm{~V}$ or $3-4.7 \mathrm{~V}$, with a current density of $17.5 \mathrm{~mA} / \mathrm{g}(\mathrm{C} / 10)$ (Figure S2). The GITT tests at multiple C-rates $(\mathrm{C} / 10, \mathrm{C} / 5$, and $\mathrm{C} / 2)$ were conducted by repetitively charging or discharging coin cells for 15 min at a current density (either $\mathrm{C} / 10, \mathrm{C} / 5$, or $\mathrm{C} / 2$ ) and recording the open-circuit potentials (OCPs) during the subsequent 5 -hour rest step.

\subsection{Operando isothermal microcalorimetry (IMC)}

A TA Instruments TAM IV multichannel isothermal microcalorimeter was used to perform the IMC experiment. The coin cell holding ampules were submerged in an oil bath in which the temperature was rigorously controlled at $30^{\circ} \mathrm{C}$ with a deviation of $\pm 0.00015{ }^{\circ} \mathrm{C}$ during $150 \mathrm{hr}$ measurements. Coin cells were connected to a BioLogic VSP potentiostat for electrochemical cycling. NMC622/Li cells were (dis)charged in potential windows of either $3-4.3 \mathrm{~V}$ or $3-4.7 \mathrm{~V}$ successively at current densities of $\mathrm{C} / 10, \mathrm{C} / 5, \mathrm{C} / 2$, and $1 \mathrm{C}$. Following each charge and discharge step, the cell potential was held until the response current drops to a $\mathrm{C} / 10$ equivalence. A 12-hour rest in open-circuit condition was introduced after all charge and discharge steps to realize the thermal equilibrium.

\section{Theory}

\subsection{Introduction}

The concept of "enthalpy potential" was firstly introduced by L. Rao and J. Newman in $1997^{[1]}$, and subsequently applied and developed by K. E. Thomas, et. $a l^{[2]}$ and G. 
Assat et. al ${ }^{[3]}$. In this work, we combined theoretical discussions on the enthalpy potential from all previous works and demonstrated the derivation of relevant equations. The physical meaning of involved variables and parameters were also elaborated.

\subsection{Equations}

We firstly consider the change rate of the total enthalpy $H$ in a battery along with time $t$. According to the law of conservation, the change rate $\left(\frac{\mathrm{d} H}{\mathrm{~d} t}\right)$ depends on the enthalpy change with the time as the only variable $\left(\frac{\partial H}{\partial t}\right)$ and the change of the control body over time (Equation (1)).

$$
\frac{\mathrm{d}}{\mathrm{d} t} \iiint_{V} \sum_{i}\left(n_{i} \overline{H_{i}}\right) d v=\iiint_{V} \sum_{i} \frac{\partial\left(n_{i} \overline{H_{i}}\right)}{\partial t} d v+\iint_{\Omega} \sum_{i}\left(n_{i} \overline{H_{i}}\right)(\vec{u} \cdot \vec{n}) d s
$$

In the Equation (1), the $n_{i}$ represents the molar density of the phase $i$, the $\overline{H_{i}}$ represents the partial molar enthalpy, the $d v$ and $d s$ are micro volume and surface area elements on the control body. The $\vec{n}$ is the normal vector of the $d s$.

The product $(\vec{u} \cdot \vec{n})$ means the velocity of the control body expansion/contraction in the normal direction. However, in a sealed electrochemical cell, we assume the macroscopic volume change of each phase could be neglected. Therefore, to understand the $\left(\frac{\mathrm{d} H}{\mathrm{~d} t}\right)$, we only need to elucidate the term $\left(\frac{\partial H}{\partial t}\right)$, namely

$$
\frac{\mathrm{d}}{\mathrm{d} t} \iiint_{V} \sum_{i}\left(n_{i} \overline{H_{i}}\right) d v=\iiint_{V} \sum_{i} \frac{\partial\left(n_{i} \overline{H_{i}}\right)}{\partial t} d v
$$

Now we analytically derive the Equation (2).

$$
\begin{aligned}
\iiint_{V} \sum_{i} \frac{\partial\left(n_{i} \overline{H_{i}}\right)}{\partial t} d v & =\iiint_{V} \sum_{i}\left(\frac{\partial n_{i}}{\partial t} \overline{H_{i}}+\frac{\partial \overline{H_{i}}}{\partial t} n_{i}\right) d v \\
& =\iiint_{V} \sum_{i}\left(\frac{\partial n_{i}}{\partial t} \overline{H_{i}}\right) d v+\iiint_{V} \sum_{i}\left(\frac{\partial \overline{H_{i}}}{\partial t} n_{i}\right) d v
\end{aligned}
$$

For the term $\iiint_{V} \sum_{i}\left(\frac{\partial \overline{H_{i}}}{\partial t} n_{i}\right) d v$ in the Equation (3), since

$$
\iiint_{V} \sum_{i}\left(\frac{\partial \overline{H_{i}}}{\partial t} n_{i}\right) d v=\iiint_{V} \sum_{i}\left\{\left(\frac{\mathrm{d} \overline{H_{i}}}{\mathrm{dT}}\right)_{p} \cdot \frac{\mathrm{d} T}{\mathrm{~d} t} \cdot n_{i}\right\} d v,
$$

so that under a rigorous isothermal condition $\left(\frac{\mathrm{d} T}{\mathrm{~d} t}=0\right)$, this term can be canceled. 
For the term $\iiint_{V} \sum_{i}\left(\frac{\partial n_{i}}{\partial t} \overline{H_{i}}\right) d v$ in the Equation (3), because the partial molar enthalpy $\overline{H_{i}}$ is independent of the molar density change rate $\frac{\partial n_{i}}{\partial t}$, we can take the $\overline{H_{i}}$ out of the integral. However, considering each phase $i$ has a different thermodynamic function $\overline{H_{i}}$, we define a relative partial molar ${\overline{H_{i}}}^{R}$ by comparing the $\overline{H_{i}}$ of each phase to a fixed phase of reference. The ${\overline{H_{i}}}^{R}$ depends on the choice of the reference.

Therefore,

$$
\begin{aligned}
\iiint_{V} \sum_{i}\left(\frac{\partial n_{i}}{\partial t} \overline{H_{i}}\right) d v & =\sum_{i}{\overline{H_{i}}}^{R} \iiint_{V}\left(\frac{\partial n_{i}}{\partial t}\right) d v \\
& =\sum_{i}{\overline{H_{i}}}^{R} \cdot \frac{\mathrm{d} N_{i}}{\mathrm{~d} t}
\end{aligned}
$$

where the $N_{i}$ represents the moles of each phase.

If the redox reaction proceeding on the target electrode is (shown as a half-reaction):

$$
a_{1} A_{1}+a_{2} A_{2}+\cdots+a_{n} A_{n}=z e^{-}
$$

where $a_{i}$ is the stoichiometric number of the phase $A_{i}$ in the reactant. $z$ moles of electrons could be transferred per mole of this reaction. Then in this reaction, according to the Faradaic law:

$$
\frac{\mathrm{d} N_{i}}{\mathrm{~d} t}=-\frac{a_{i} I}{z F}
$$

It is worth noting that by applying the Faradaic law, we assume Faradaic processes are the sole reason of electroative material generation or consumption. So any parasitic reaction in a real battery system that could potentially introduce extra phase transitions is neglected.

Herein, by neglecting tiny current heterogeneity in local electrode areas, we assume that when a current $I$ is applied to the cell, the same current flux flows through each phase. By combining the Equation(2), Equation(3), Equation(4), and Equa$\operatorname{tion}(6)$

$$
\begin{aligned}
\frac{\mathrm{d} H}{\mathrm{~d} t} & =\frac{\mathrm{d}}{\mathrm{d} t} \iiint_{V} \sum_{i}\left(n_{i} \overline{H_{i}}\right) d v \\
& =-\sum_{i}{\overline{H_{i}}}^{R}\left(\frac{a_{i} I}{z F}\right)
\end{aligned}
$$

From the first law of the thermodynamics, if the system has constant volume and pressure,

$$
d H=(d U)_{v, p}=d Q-d W
$$

In an eletrochemical cell, we consider the work done by the system is electrical work, 
namely $d W=(I E) d t$, then based on Equation (8):

$$
\frac{\mathrm{d} H}{\mathrm{~d} t}=\frac{\mathrm{d} Q}{\mathrm{~d} t}-I E
$$

Combining the Equation (7) and Equation (9),

$$
\begin{aligned}
\dot{Q}-I E & =-\sum_{i}{\overline{H_{i}}}^{R}\left(\frac{a_{i} I}{z F}\right) \\
& =-I\left(\frac{\Delta H}{z F}\right)
\end{aligned}
$$

In the Equation (10), the $\Delta H$ represents the total enthalpy change summed by all phases. Because the relative partial molar enthalpy of each phase takes the same reference, so the sum $\Delta H=\sum_{i}\left(a_{i} \bar{H}_{i}{ }^{R}\right)$ cancels the relativity. The thermodynamic term in the Equation 8 can be defined as a new parameter: ethalpy potential $\left(U_{H}\right)$ (Equation (11)).

$$
U_{H}=-\frac{\Delta H}{z F}
$$

So that the Equation (10) can be written as:

$$
U_{H}=E-\frac{\dot{Q}}{I}
$$

In the electrochemical system described in this context (shown as Equation (5)), the gibbs free energy change is:

$$
\Delta G=-z F E_{e q}
$$

From the isothermal gibbs free energy relation $\Delta G=\Delta H-T \Delta S$, we could determine the entropy change:

$$
\Delta S=\frac{1}{T}(\Delta H-\Delta G)
$$

By substituting Equation (11) and Equation (13) into the Equation (14), the $\Delta S$ can be expressed as:

$$
\Delta S=\frac{z F}{T}\left(E_{e q}-U_{H}\right)
$$

\subsection{Discussion}

From the Equation (9) to Equation (11), we notice that:

$$
\frac{\mathrm{d} H}{\mathrm{~d} t}=I \cdot U_{H}
$$


The Equation (16) reveals the nature of the parameter $U_{H}$. When a current $I$ is applied to this system, the enthalpy potential reflects the change rate of the total enthalpy over time.

Interestingly, the $U_{H}$ has a dimension of voltage (in a unit of volt). According to the Equation (15), the difference between the $U_{H}$ and the thermodynamic potential $E_{e q}$ determines the magnitude of entropy change in the system.

From the Equation (12), it is easy to obtain:

$$
\dot{Q}=I\left(E-U_{H}\right)
$$

If we regard the parameter $U_{H}$ as an adjusted thermodynamic equilibrium potential giving consideration to the entropy change in the system, the Equation (17) applies to the form of Ohm's law. The term $\left(E-U_{H}\right)$ could be viewed as effective polarization. Therefore, once the $U_{H}$ of an electrochemical cell is determined, the heat generation of a battery could be simplified as ohmic heat. Moreover, the enthalpy potential is a pure thermodynamic property. To study heat generation at different rates, if the electrochemical process does not change due to kinetic factors, then the enthalpy potential $U_{H}$ is also constant, and the current $I$ becomes the only kinetic factor to consider. Introducing the concept of $U_{H}$ could thereby simplify the thermal modeling and management of batteries significantly by treating them as equivalent resistors.

\section{Supplemental Results}

\subsection{The characterization of NMC622 electrodes}

(a)

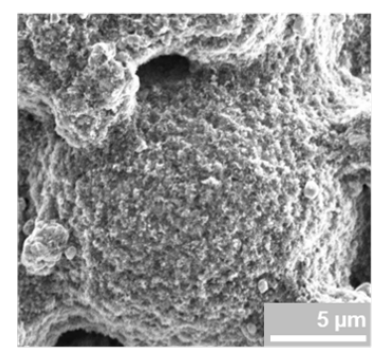

(b)

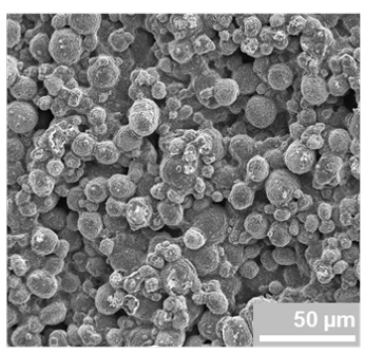

(c)

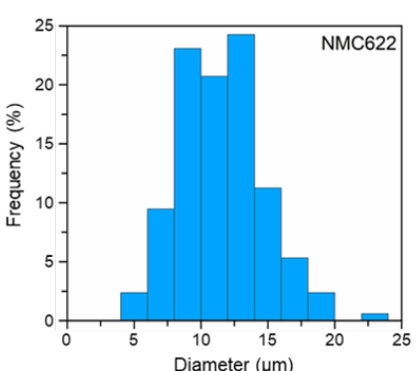

Figure S1. The characterization of NMC622 electrodes. SEM images of a NMC622 pristine coating at (a) 5000X and (b) 500X magnification. (c) Particle size distribution as determined by SEM. 


\subsection{Electrochemistry of formation cycles}

(a)

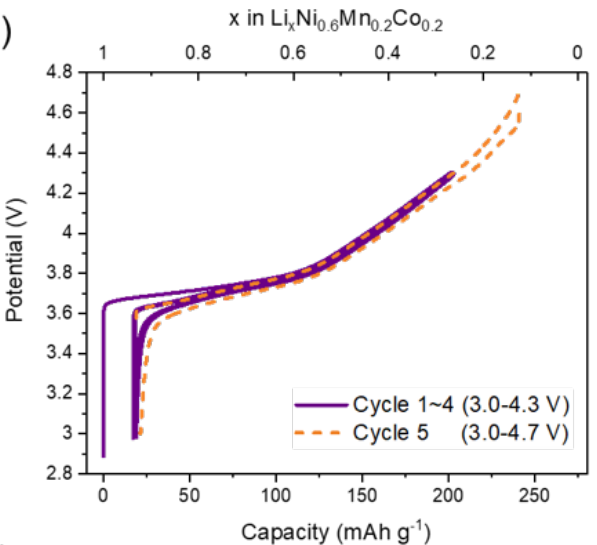

(c)

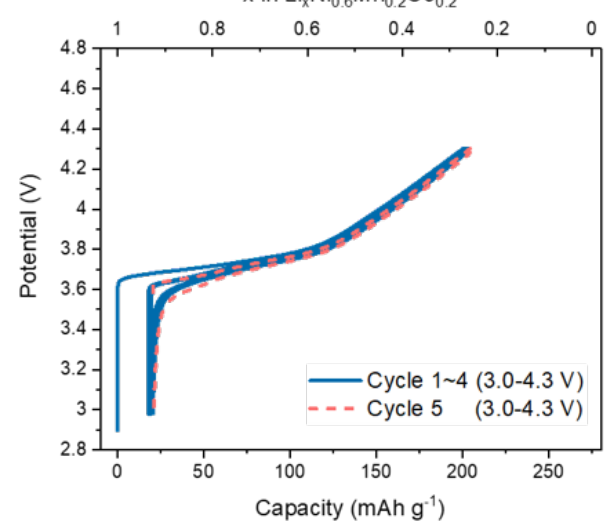

(b)

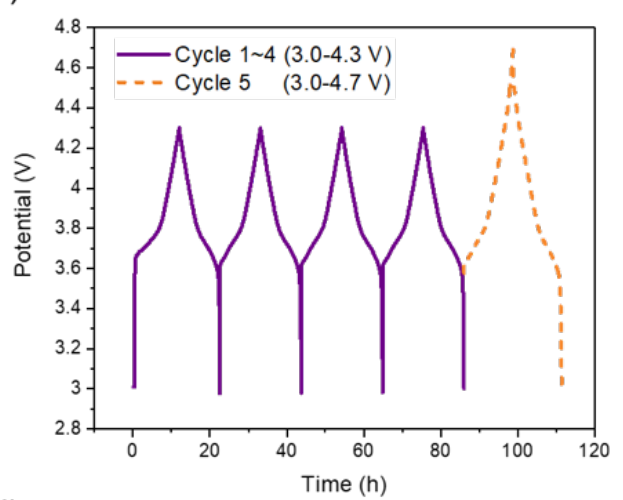

(d)

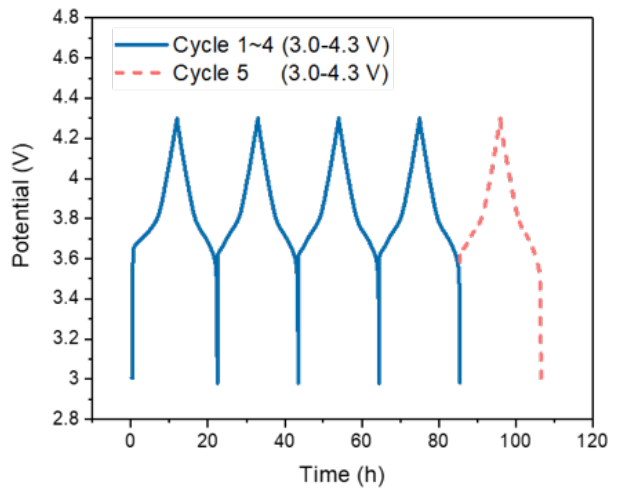

Figure S2. Electrochemistry of formation cycles of cells prepared for the IMC tests in potential windows of (a), (b) 3.0-4.7 V and (c), (d) 3.0-4.3 V. For the IMC tests in 3.0-4.7 V, cells were pre-cycled in 3.0-4.3 V for 4 cycles and 3.0-4.7 $\mathrm{V}$ for one cycle. For the IMC tests in 3.0-4.3 V, all 5 pre-IMC cycles were in 3.0-4.3 V. 


\subsection{The duplicate IMC tests}

(a)

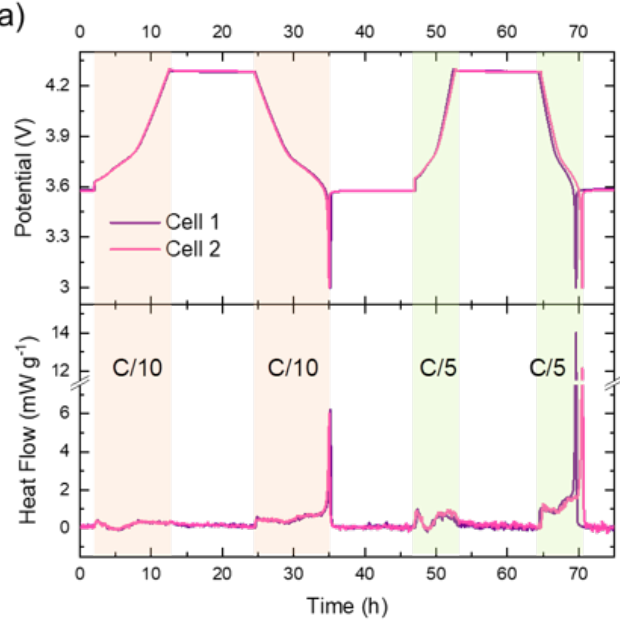

(b)

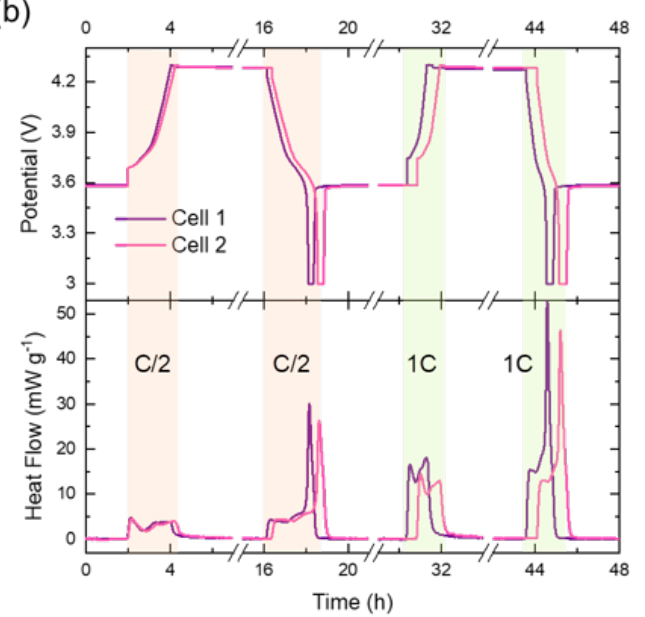

Figure S3. The duplicate IMC test for cell cycled in 3.0-4.3 V at multiple rates of current: (a) at C/10 and $\mathrm{C} / 5$, (b) at $\mathrm{C} / 2$ and $1 \mathrm{C}$.

(a)

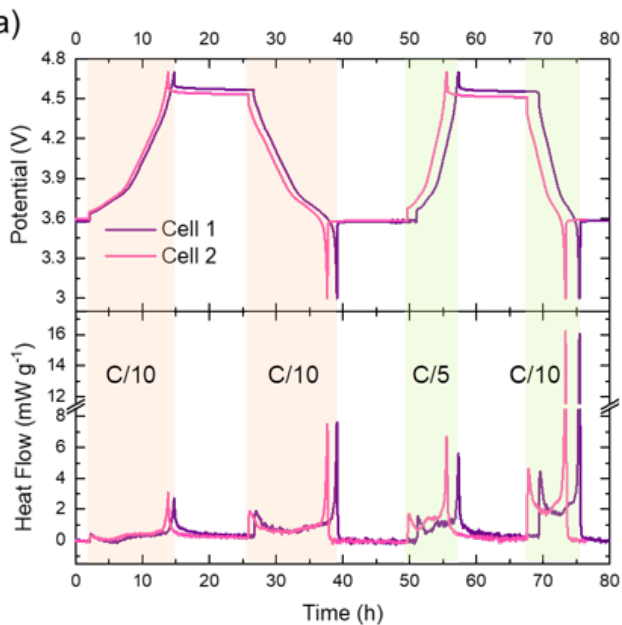

(b)

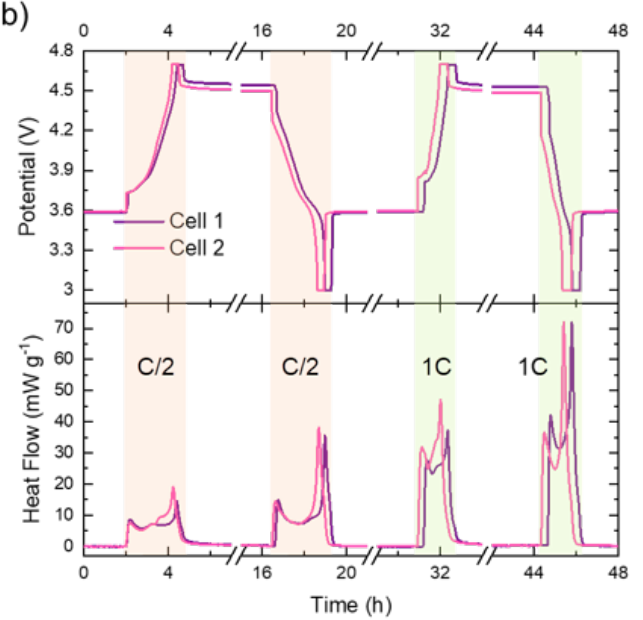

Figure S4. The duplicate IMC test for cell cycled in 3.0-4.7 V at multiple rates of current: (a) at C/10 and $\mathrm{C} / 5$, (b) at $\mathrm{C} / 2$ and $1 \mathrm{C}$. 


\subsection{Operando IMC tests: Electrochemistry and real-time heat flow}

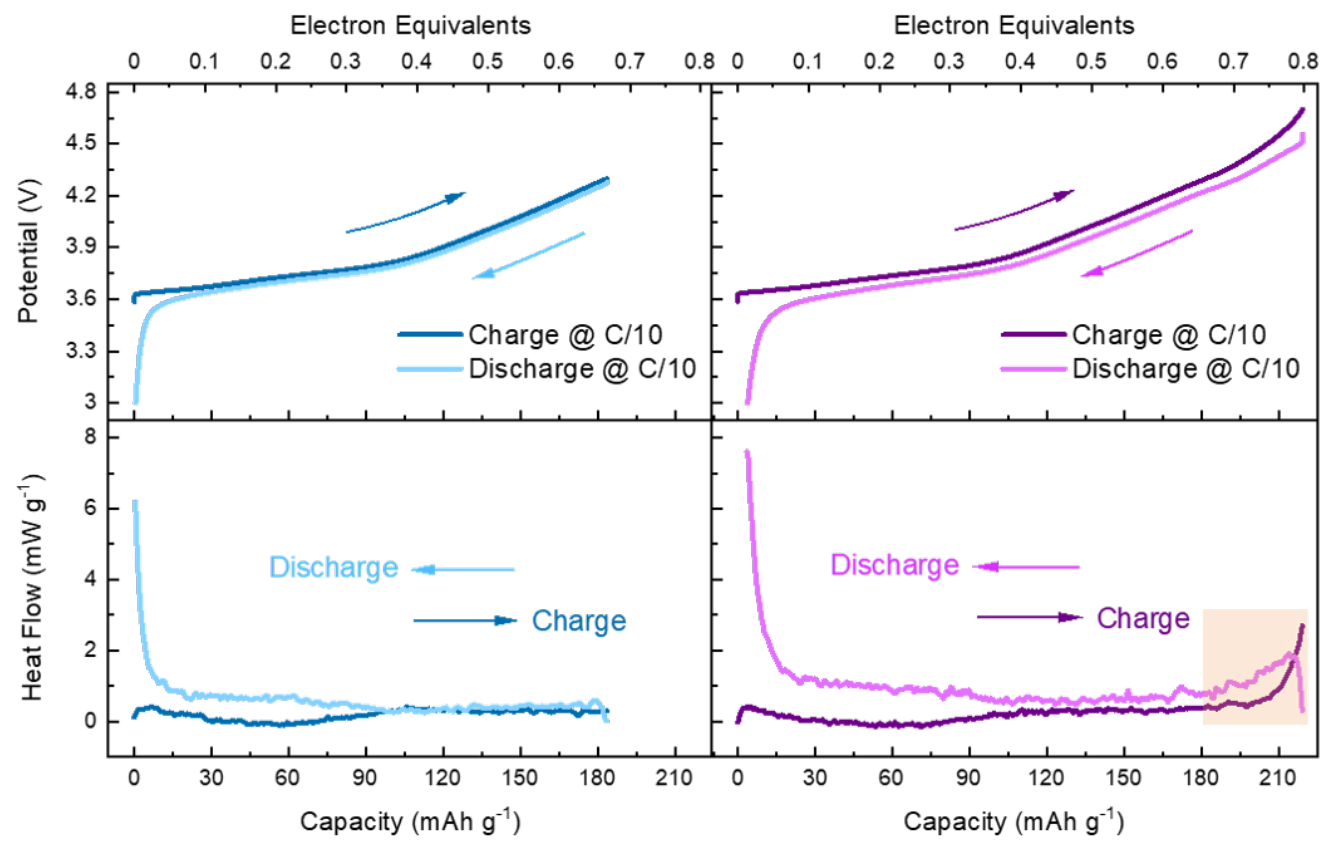

Figure S5. Operando IMC tests in two potential ranges at a current density of C/10.

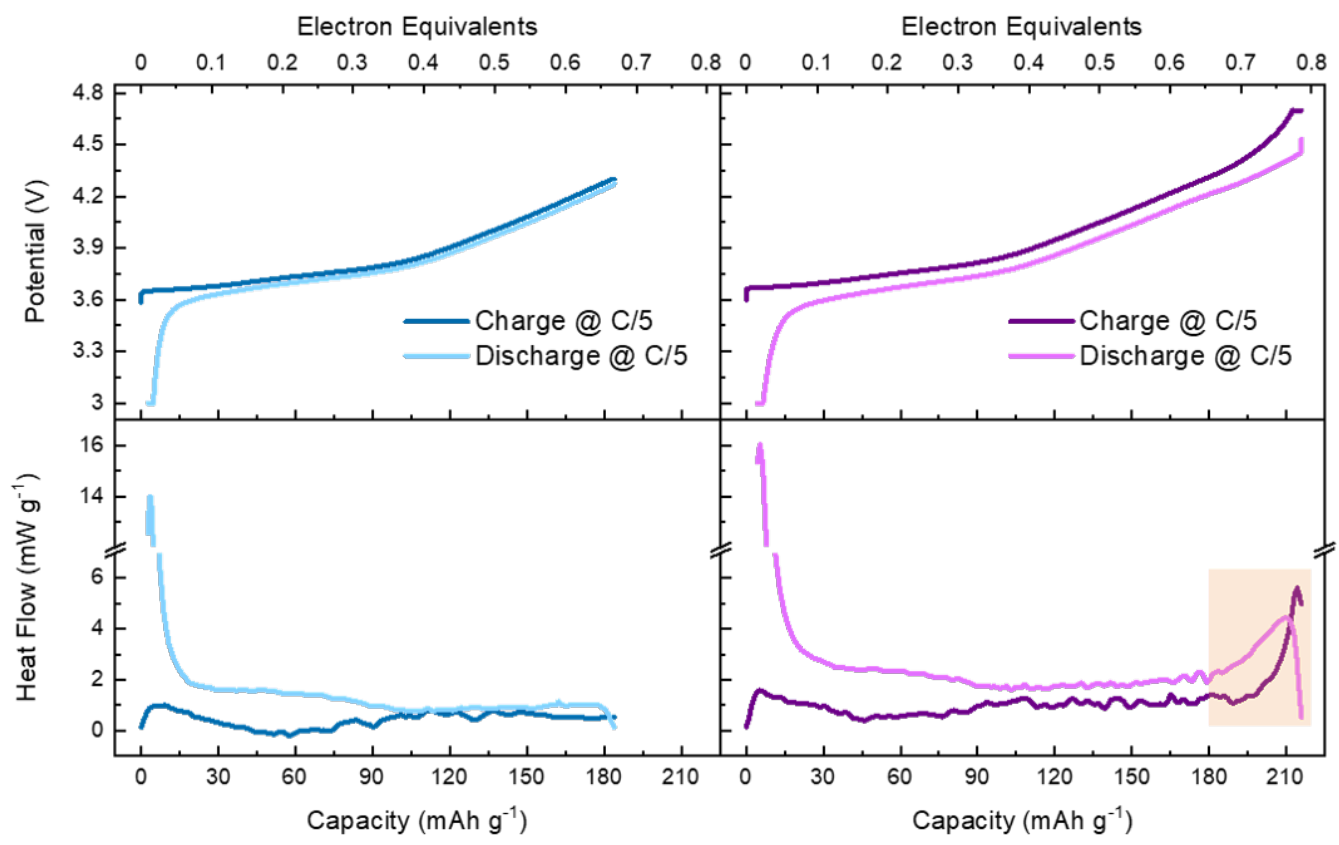

Figure S6. Operando IMC tests in two potential ranges at a current density of $\mathrm{C} / 5$. 


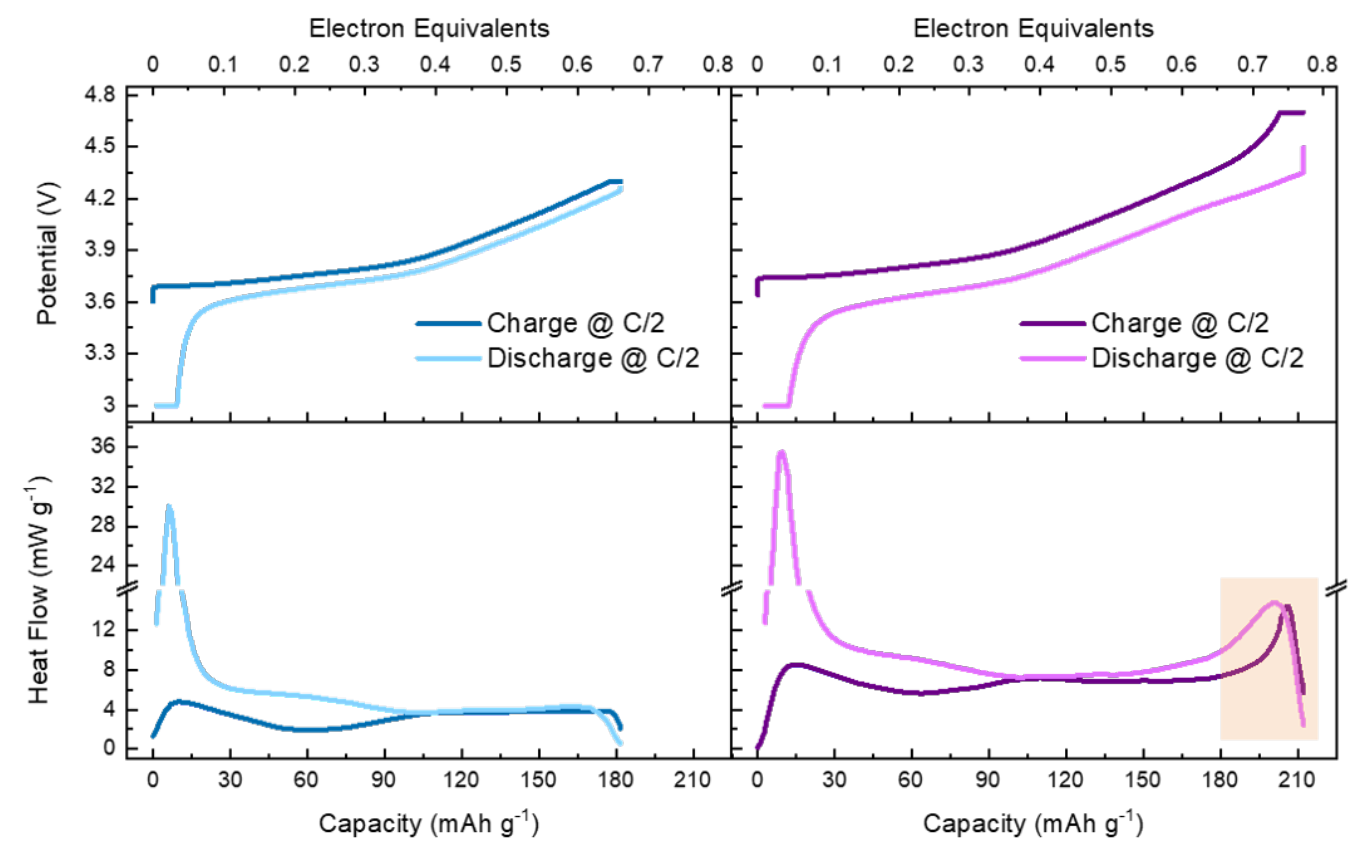

Figure S7. Operando IMC tests in two potential ranges at a current density of $\mathrm{C} / 2$.

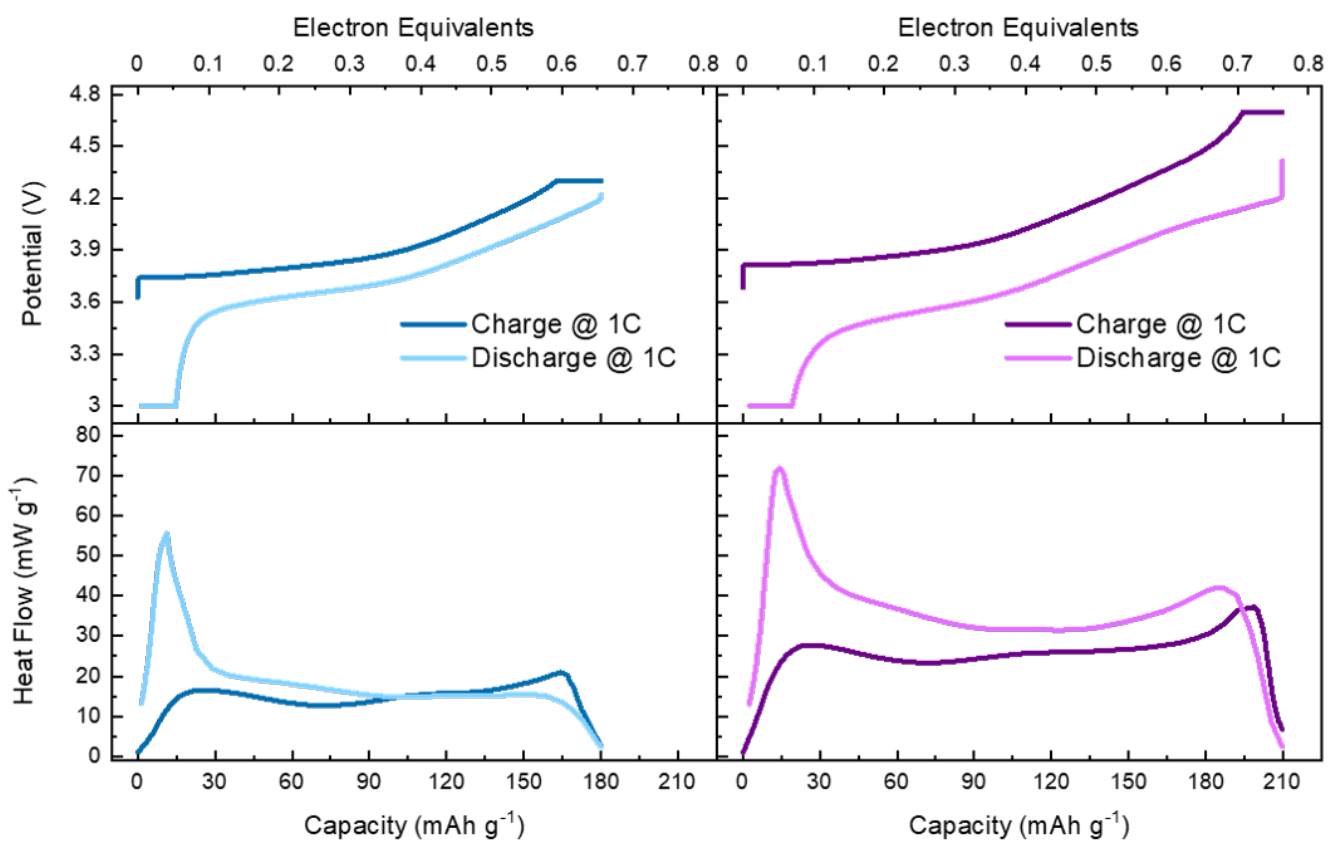

Figure S8. Operando IMC tests in two potential ranges at a current density of 1C. 


\subsection{The estimation of thermodynamic equilibrium potential by GITT}

(a)

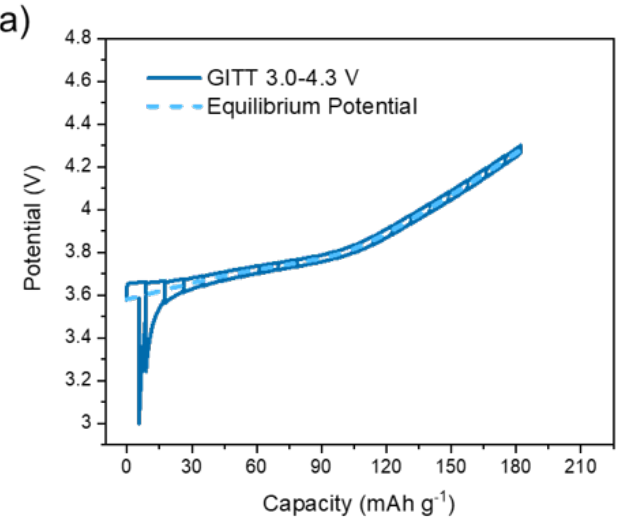

(c)

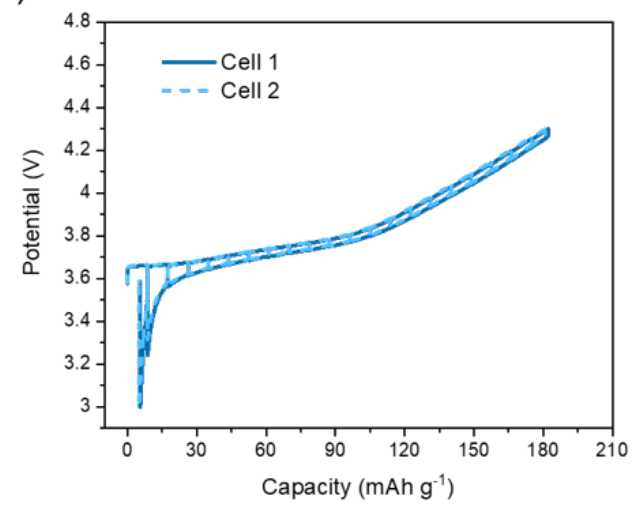

(b)

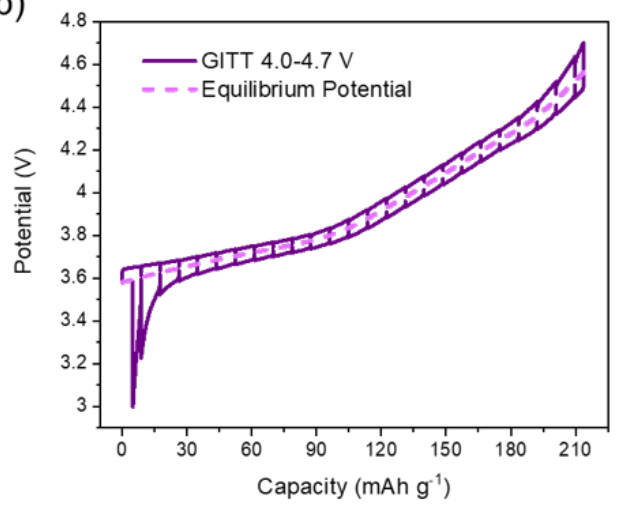

(d)

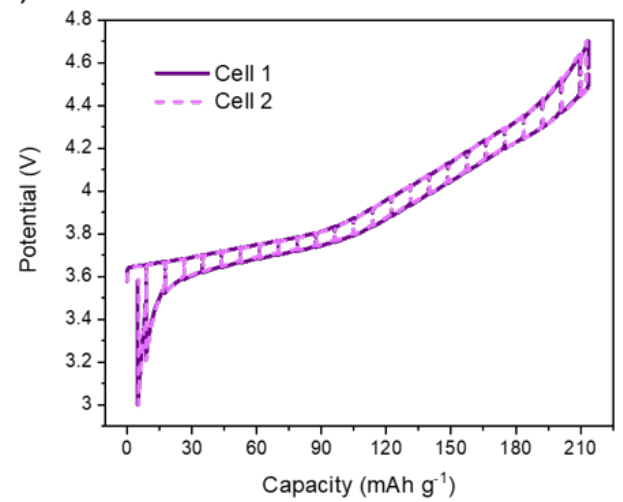

Figure S9. Galvanostatic intermittent titration technique (GITT) tests in the potential range of (a) 3.0-4.3 $\mathrm{V}$ and (b) 3.0-4.7 V at a C-rate of C/5. The equilibrium potentials, shown as dashed lines in (a) and (b), were estimated by taking the average value of OCPs after 5-hour rest following charge and discharge steps at the same state of charge. The "thermodynamic equilibrium" is technically a different concept from "open-circuit state". Duplicate GITT tests were shown in (c) and (d) to demonstrate the reproducibility of the GITT test. 


\subsection{Potentiometric measurement of entropy}

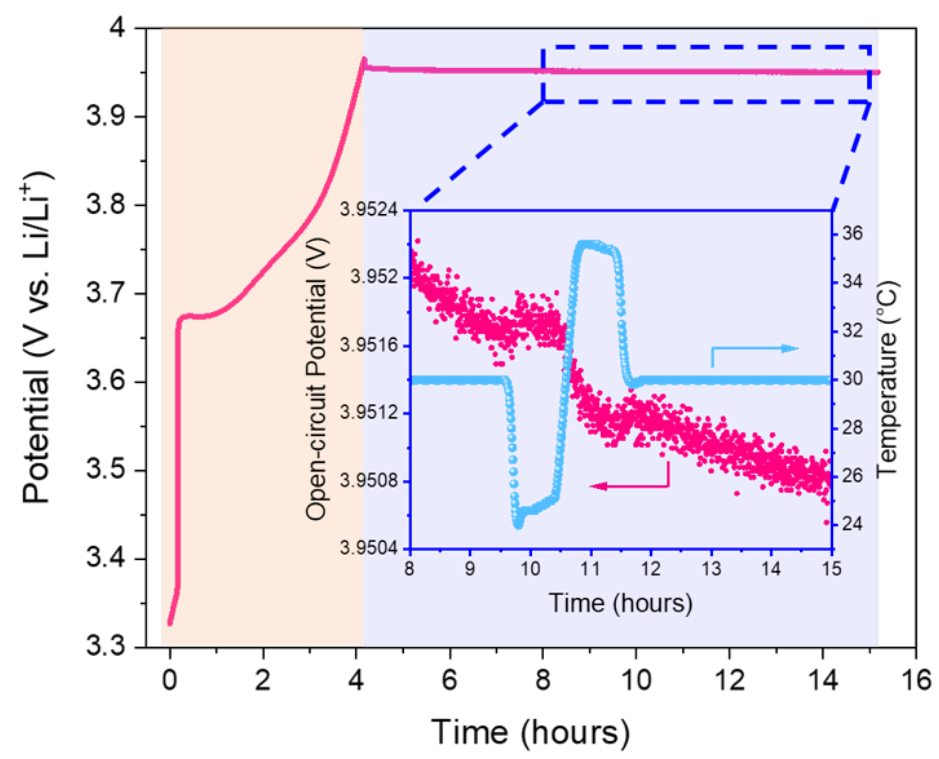

Figure S10. Potentiometric measurement of entropy. The cell was charged at C/5 for 4 hours reaching a state of charge of approximately $80 \%$ and then kept at the open-circuit condition for 5 hours at a constant temperature of $30{ }^{\circ} \mathrm{C}$. The ambient temperature was then controlled in a sequence of $30{ }^{\circ} \mathrm{C}-25^{\circ} \mathrm{C}-35^{\circ} \mathrm{C}$ - $30^{\circ} \mathrm{C}$. The open-circuit potential (OCP) was recorded upon the temperature variation. The OCP evolution triggered by the temperature change was insignificant. Therefore, according to the equation $\Delta S=z F\left(\frac{\partial E_{o c p}}{\partial T}\right)$ ${ }^{[4]}$, the entropy change $\Delta S$ is small.

\subsection{Electrochemistry shown as chemical composition}

(a)

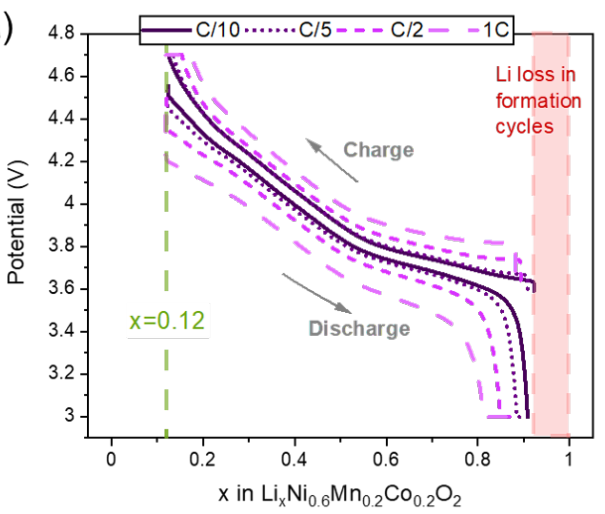

(b)

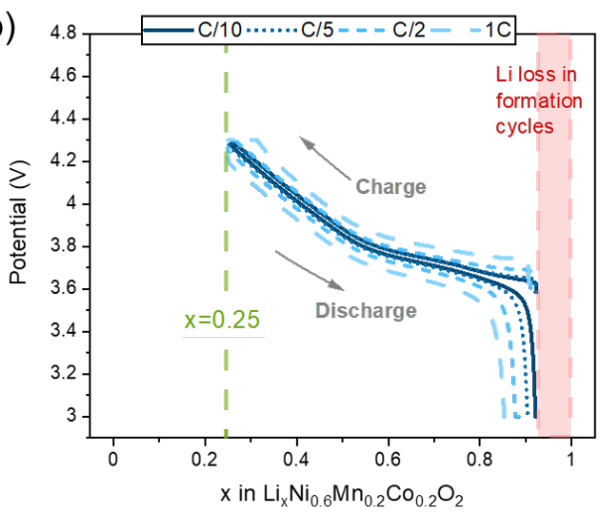

Figure S11. Electrochemistry of cells tested in the IMC in potentials of (a) 3.0-4.7 V and (b) 3.0-4.3 V shown as the chemical composition of $\mathrm{Li}_{x} \mathrm{Ni}_{0.6} \mathrm{Mn}_{0.2} \mathrm{Co}_{0.2} \mathrm{O}_{2}$ 


\subsection{Normalized heat generated per unit of capacity}

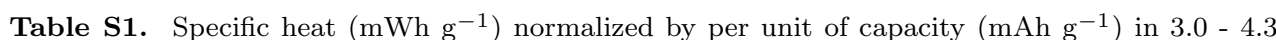
V. The "charge" and "discharge" numbers were calculated by taking specific heat in charge (or discharge) divided by capacities. The value of "(dis)charge averaged" is calculated by taking the arithmetic mean value of normalized heat values of charge and discharge.

\begin{tabular}{lccc}
\hline & Charge $(\mathrm{mWh} / \mathrm{mAh})$ & Discharge $(\mathrm{mWh} / \mathrm{mAh})$ & $($ Dis $)$ charge Averaged $(\mathrm{mWh} / \mathrm{mAh})$ \\
\hline $\mathrm{C} / 10$ & 0.011 & 0.034 & 0.023 \\
$\mathrm{C} / 5$ & 0.013 & 0.045 & 0.029 \\
$\mathrm{C} / 2$ & 0.038 & 0.124 & 0.081 \\
$1 \mathrm{C}$ & 0.093 & 0.239 & 0.166 \\
\hline
\end{tabular}

Table S2. Specific heat $\left(\mathrm{mWh} \mathrm{g}^{-1}\right)$ normalized by per unit of capacity $\left(\mathrm{mAh} \mathrm{g}^{-1}\right)$ in $3.0-4.7 \mathrm{~V}$.

\begin{tabular}{lccc}
\hline & Charge $(\mathrm{mWh} / \mathrm{mAh})$ & Discharge $(\mathrm{mWh} / \mathrm{mAh})$ & $($ Dis $)$ charge Averaged $(\mathrm{mWh} / \mathrm{mAh})$ \\
\hline $\mathrm{C} / 10$ & 0.015 & 0.060 & 0.037 \\
$\mathrm{C} / 5$ & 0.034 & 0.077 & 0.056 \\
$\mathrm{C} / 2$ & 0.090 & 0.139 & 0.115 \\
$1 \mathrm{C}$ & 0.165 & 0.257 & 0.211 \\
\hline
\end{tabular}

\section{References}

[1]. Rao, L. and Newman, J., Heat-Generation Rate and General Energy Balance for Insertion Battery Systems. J. Electrochem. Soc., 1997, 144(8), 2697.

[2]. Thomas, K.E. and Newman, J., Thermal Modeling of Porous Insertion Electrodes. J. Electrochem. Soc., 2003, 150(2), A176.

[3]. Assat, G., Glazier, S.L., Delacourt, C. and Tarascon, J.M., Probing the Thermal Effects of Voltage Hysteresis in Anionic Redox-Based Lithium-Rich Cathodes Using Isothermal Calorimetry. Nat. Energy, 2019, 4(8), 647.

[4]. Zhang, X.F., Zhao, Y., Patel, Y., Zhang, T., Liu, W.M., Chen, M., Offer, G.J. and Yan, Y., Potentiometric Measurement of Entropy Change for Lithium Batteries. Phys. Chem. Chem. Phys., 2017, 19(15), 9833. 